\title{
Reconfiguración territorial y estrategias de reproducción social en el periurbano poblano*
}

José Álvaro Hernández Flores ${ }^{* *}$

Beatriz Martínez Corona ${ }^{* * *}$

José Arturo Méndez Espinoza****

Recibido: 2013-06 -20 Aprobado: 2013-II-15 Disponible en línea: 2014-07-27

doi:Io.III44/javeriana.CRDII-74.rter

Cómo citar este artículo: Hernández Flores, J. A., Martínez Corona, B. y Méndez Espinoza, J. (2014).

Reconfiguración territorial y estrategias de reproducción social en el periurbano poblano. Cuadernos de Desarrollo

Rural, ${ }_{I I}(74)$, I3-34. http://dx.doi.org/I0.III44/Javeriana.CRDII-74.rter

\section{Resumen}

Mediante el análisis de las estrategias de reproducción desplegadas por los grupos domésticos periurbanos de San Diego Cuachayotla (Puebla, México), el artículo examina las diversas modalidades bajo las cuales se recrea lo social en espacios rurales sometidos a tensiones ocasionadas por la interacción con procesos y actores de otros ámbitos. Asimismo se analiza el papel que desempeñan las prácticas agrícolas como parte de estas estrategias. Entre los resultados del estudio de caso destaca la descripción de la forma como ocurre el proceso de periurbanización en territorios con un fuerte componente rural, que da lugar a la formulación de estrategias de reproducción específicas, derivadas del empalme o superposición de lo urbano, con expresiones determinadas, muy concretas, de lo rural.

\section{Palabras clave:}

estrategias de reproducción; grupo doméstico; nueva ruralidad; periurbanización; Cholula (México)

*El artículo es una revisión o síntesis del proyecto Modo de vida, estrategias reproductivas y reconfiguración territorial en la región conurbada del centro de México, financiado por el Consejo Nacional de Ciencia y Tecnología.

**Doctor en Ciencias con Especialidad en Estrategias para el Desarrollo Agrícola Regional, profesor en la Facultad de Economía, Benemérita Universidad Autónoma de Puebla. Correo electrónico: josealvarohf@gmail.com

${ }^{* * *}$ Doctora en Ciencias con Especialidad en Estrategias para el Desarrollo Agrícola Regional, profesorainvestigadora de tiempo completo. Colegio de Postgraduados. Correo electrónico: beatrizm@colpos.mx *****Doctor en Geografía, profesor-investigador de tiempo completo. Colegio de Postgraduados.

Correo electrónico: jamendez@colpos.mx

\section{cc) 0}




\title{
Territorial Reconfiguration and Strategies of Social Reproduction in the Poblano Peri-Urban
}

\begin{abstract}
By analyzing the reproduction strategies deployed by the peri-urban domestic groups from San Diego Cuachayotla (Puebla, Mexico), the article examines the various forms under which the social sphere is recreated in rural areas under stress caused by the interaction of processes and actors from other areas. It is also analyzed the role of agricultural practices as part of these strategies. Among the results of the case study, it stands out the description of how the peri-urbanisation process occurs in territories with a strong rural component and it leads to the formulation of specific breeding strategies derived from the joint or overlapping of the urban sphere with certain specific expressions of what rural is.
\end{abstract}

Keywords:

reproduction strategies; domestic group; new rurality; peri-urbanisation; Cholula.

\section{Reconfiguration territoriale et stratégies de reproduction sociale dans la zone périurbaine de Puebla, Mexique}

\section{Résumé}

À travers de l'analyse des stratégies de reproduction déployées par les groupes domestiques périurbains de San Diego Cuachayotla (Puebla, Mexique), l’article examine les différentes modalités sous lesquelles se conçoit la partie sociale dans des espaces ruraux soumis à des tensions causées pour l'interaction avec des processus et des participants d'autres milieux. De même, le rôle des pratiques agricoles s'analyse comme une partie des ces stratégies. Parmi les résultats de l'étude de cas il faut mettre en relief la description de la manière dans laquelle le processus de périurbanisation se passe dans des territoires qui ont une forte composante rurale, et qui donne lieu à la formulation de stratégies de reproduction spécifiques, conçues grâce à l'enchaînement ou à la superposition de ce qui est urbain, avec des expressions déterminées, bien concrètes, de ce qui est rural.

Mots-clés auteurs:

stratégies de reproduction; groupe domestique; nouveau ruralité; périurbanisation; Cholula (Mexique) 


\section{Introducción}

En las últimas décadas, el crecimiento desordenado y acelerado de las ciudades ha impactado de manera significativa la estructura de los territorios ubicados a lo largo de su periferia. El resultado de este proceso ha sido la formación de espacios periurbanos, caracterizados por mantener una situación intermedia entre lo urbano y lo rural, los cuales están expuestos a una serie de cambios demográficos, económicos e institucionales que suscitan una gama muy diversa de respuestas a escala local (Banzo, 2005; Barsky, 2005; Entrena, 2005). La constitución de estos espacios suele ir aparejada de la fragmentación territorial de los procesos productivos locales, así como de cambios importantes en el modo de vida, configuración identitaria y prácticas sociales, resultado de la adaptación de los actores a los nuevos contextos (Ávila, 200I).

Existe una gran diversidad de estudios, de corte urbanista, donde la dinámica de los espacios periurbanos se analiza a partir del proceso de expansión de la ciudad y la consecuente transformación de las áreas rurales que la circundan. En este tipo de aproximaciones, el periurbano se concibe como un territorio que está expuesto a la presión urbana y que es susceptible de ser ocupado, ya que desde la perspectiva de la ciudad se le considera generalmente como un área de reserva territorial (Ávila, 2004). Subyace bajo este enfoque, la idea de "urbanización de lo rural”, entendida como una tendencia "natural", irrefrenable, consecuencia de los procesos de concentración urbana y movilidad poblacional propios de la vida moderna'.

No es raro que a partir de este tipo de conceptualizaciones, muchos autores coincidan en visualizar al periurbano como un espacio multifuncional, sometido a transformaciones profundas y veloces, cuyo dinamismo está en gran medida determinado por el crecimiento de la ciudad y su paulatina incorporación a la jerarquía del conjunto urbano (Entrena, 2005).

No obstante, existen otro tipo de aproximaciones que privilegian una perspectiva más afín con la estructuración y dinámica de los territorios rurales, y que abordan el estudio de las relaciones campo-ciudad en los espacio periurbanos desde una óptica más rural que urbana (Galimberti, 20II). Este tipo de enfoques, en los que predomina la idea de la "ruralización de lo urbano", permiten conocer el fenómeno desde una dimensión en la cual cobran importancia, tanto las transformaciones

I La concentración urbana, en tanto esquema de organización territorial que alude al carácter denso de la relación entre población por unidad de área, es la expresión espacial dominante de las sociedades contemporáneas. Lo mismo puede decirse de la movilidad poblacional, que se refiere al proceso de expansión del hábitat urbano sobre el medio rural, así como a traslados diarios entre domicilio y espacio de trabajo. 
que experimentan los espacios rurales que están en contacto con la urbe, como las situaciones socioespaciales que se derivan y que experimentan de forma cotidiana los actores rurales que habitan en estos espacios (Delgado, 2003).

Al tomar distancia de las lecturas polares y dicotómicas, este tipo de posturas permite entender cómo se modelan los nuevos territorios, cómo inciden los cambios en el sistema productivo local y cuáles son las funciones que asumen los espacios rurales ante las transformaciones inducidas por el contexto urbano. Esto último resulta particularmente útil al analizar las estrategias de reproducción social de los grupos domésticos periurbanos, en el contexto de los cambios demográficos, económicos e institucionales que han impactado a lo largo de las últimas décadas a las sociedades rurales (Pérez, 200I).

Estos cambios se evidencian en fenómenos tales como la desagrarización del mundo rural; reducción progresiva del aporte de las actividades agrícolas a la generación de ingreso en el campo; incremento de la pluriactividad, que en los contextos campesinos se expresa en una compleja combinación entre actividades agropecuarias y asalariadas que coexisten con pequeños negocios y oficios propios; la intensificación de los procesos migratorios; y la feminización de la agricultura, entre otros (Hernández y Méndez, 2007).

Diversos autores (Kay, 2004; Arias, 2006; Ramírez, 2006) advierten que la paulatina pérdida de centralidad de la agricultura -fenómeno que se manifiesta con particular intensidad en los espacios periurbanos- puede llegar a traducirse en planteamientos discursivos donde las actividades no agrícolas se conciben como una etapa en el desarrollo de las economías rurales y no como resultado de la crisis y abandono del sector primario por parte del Estado; lo anterior, alimenta una visión en que los campesinos no tienen posibilidades de reproducirse como productores de alimentos básicos, por lo que deben insertarse en otro tipo de actividades (Rubio, 2006).

Al respecto, es necesario señalar que si bien la pérdida relativa de significación económica y social de las actividades primarias en las sociedades rurales está plenamente documentada (De Janvry y Sadoulet, 200I; Dirven, 2004; Klein, I992; Reardon, Berdegué y Escobar, 200I), en muchas regiones del mundo los procesos agrícolas urbanos y periurbanos constituyen un componente importante en la economía familiar. Por tanto, este tipo de prácticas deben ser valoradas en términos de sus aportaciones a la seguridad alimentaria y como un componente central de las estrategias económicas de la población que habita en la periferia de las ciudades (FAO, I999; Hernández, 2010). 
Asimismo, en muchas regiones periurbanas o conurbanas ${ }^{2}$ el modo de vida continúa siendo campesino, no porque la agricultura conserve la misma significación económica, sino porque son los referentes identitarios y comunitarios tradicionales, propios de las sociedades rurales, los que conceden coherencia a las prácticas sociales (Pérez, 2005; Navarro, 2005).

El presente artículo pretende, mediante la caracterización de las estrategias de reproducción social que desarrollan los grupos domésticos de una localidad periurbana, identificar el papel que desempeñan las prácticas agrícolas como parte de esas estrategias, al tiempo de examinar las diversas modalidades bajo las cuales se recrea lo social en espacios sometidos a tensiones derivadas de la interacción con procesos y actores provenientes de otros ámbitos. La hipótesis que guía este trabajo es que incluso en un contexto de profundos cambios y transformaciones territoriales, las prácticas sociales y productivas asociadas con el modo de vida rural mantienen un papel central en el desarrollo de estrategias orientadas a la reproducción social de los grupos domésticos periurbanos.

\section{Metodología}

La localidad que se seleccionó como estudio de caso fue la de San Diego Cuachayotla, junta auxiliar del municipio conurbado de San Pedro Cholula. Esta localidad, con antecedentes rurales, acusa desde hace varias décadas un acelerado proceso de urbanización como resultado de su cercanía física con la capital del estado de Puebla, en la región centro de México. A lo largo de la investigación se privilegió el uso de técnicas de investigación cualitativa que permitieran analizar, interpretar y comprender las estrategias de reproducción de los grupos domésticos, al tiempo de expresar la interrelación existente entre sus condiciones de vida y los significados subjetivos que sus integrantes otorgan a las mismas.

En este sentido, las principales técnicas que se utilizaron fueron: el análisis de contenido, la observación participante y la entrevista en profundidad. En total, se

2 Conviene distinguir entre los procesos de urbanización, conurbación y periurbanización. En tanto que el primero alude al crecimiento físico de las áreas urbanas; el segundo se refiere a la integración espacial de estas áreas en un solo sistema, pero bajo la misma independencia funcional y dinámica. La periurbanización, por su parte, su utiliza para describir el proceso de difusión urbana y transformación espacial del medio rural en la periferia de las grandes ciudades. 
llevaron a cabo diez entrevistas en profundidad -realizadas entre 2008 y 2009- así como una extensa revisión bibliográfica, hemerográfica y estadística, para indagar cuestiones vinculadas con la posición socioeconómica de los grupos domésticos, sus características sociodemográficas, los cambios en la estructura productiva local, entre otros aspectos relevantes que se describen a continuación.

\section{San Diego Cuachayotla, ¿una localidad rural?}

En los últimos años, la ciudad de Puebla -ubicada a Izo kilómetros de la capital de México- ha experimentado un crecimiento urbano explosivo y desordenado, sustentado en la incorporación de territorios que históricamente se han caracterizado por poseer una vocación agrícola. Parte de este crecimiento ha tenido lugar sobre los municipios que se ubican a lo largo de la carretera que une a este estado con la capital del país.

Ubicada a un kilómetro del centro de la cabecera municipal de San Pedro Cholula, resulta casi imposible distinguir las fronteras que separan a San Diego Cuachayotla de las poblaciones que la rodean. La mancha urbana en esta zona del municipio ha desdibujado por completo los límites entre esta localidad y los pueblos que la circundan. Un dato puede resultar relevante para entender el grado en que esta localidad ha sido alcanzada por la urbe: hasta 1980 San Diego Cuachayotla aparecía en los censos del Instituto Nacional de Estadística y Geografía (INEGI) bajo la categoría de "pueblo".

Sin embargo, para la década de los años noventa desapareció de las estadísticas oficiales. La razón, de acuerdo con las autoridades de INEGI, es que dada su cercanía con la cabecera municipal, San Diego Cuachayotla fue recategorizada como "localidad conurbana”, por lo que muchos de los datos estadísticos que antes se encontraban disponibles de manera exclusiva para ese centro poblacional, aparecen agregados en el censo del año 2000, como parte de la cabecera municipal de Cholula de Rivadavia.

Peor aun, para el censo de 2010, los pocos indicadores que existían agregados para esta localidad desaparecieron. La anécdota no es menor en cuanto refleja una situación evidente a los ojos de cualquiera que intente acercarse a la realidad económica y social de esta localidad. Y es que en términos estadísticos, a los habitantes de San Diego literalmente "se los comió" la ciudad. Aunque para fines políticos y administrativos San Diego Cuachayotla conserva dentro de la estructura jerárquica municipal su categoría de junta auxiliar, al menos para el INEGI pasó de ser considerado como un pueblo, a ser una colonia más adscrita a la cabecera. 
La junta auxiliar de San Diego Cuachayotla ocupa una extensión aproximada de tres kilómetros cuadrados, donde habita un total de 6697 personas (INEGI, 2010). El paisaje que predomina en esta junta auxiliar constituye una mezcla de elementos tradicionales -que remiten de modo inevitable a un estilo de vida apegado a la ruralidad-, y otros de carácter "moderno", que rinden cuenta de las transformaciones recientes inducidas por la cercanía con los centros urbanos.

En el caso de San Diego Cuachayotla destacan como elementos paisajísticos e identitarios vinculados a un modo de vida rural los siguientes: algunas porciones del territorio destinadas a la agricultura de autosubsistencia; la formación de la vivienda tradicional campesina, que reserva el traspatio para el cultivo del maíz y la cría de animales; y la persistencia de actividades religiosas y festivas sustentadas en instituciones comunitarias de origen campesino, como la cooperación o el sistema de cargos.

Por otra parte, entre los elementos vinculados con una supuesta modernidad, se pueden mencionar la infraestructura y la dotación de servicios públicos, tales como alumbrado, seguridad, recolección de basura, drenaje y pavimentación, presentes en el primer cuadro de la localidad y calles aledañas. Asimismo, resulta evidente a simple vista la alteración del paisaje impuesta por la fabricación de ladrillo, actividad principal de esta localidad, la cual demanda el uso de cierto tipo de edificaciones, como son: las galeras para cortar el barro, los hornos para cocer el ladrillo, los depósitos de petróleo y grandes áreas abiertas donde se ponen a secar los bloques antes de cocerlos. Todos estos elementos le confieren un ambiente característico a San Diego Cuachayotla, sobre todo porque se ubican en los predios urbanos, y forman una sola unidad con las construcciones de la casa-habitación y el traspatio.

Si se entiende el paisaje como "el arreglo que históricamente forman los objetos en el espacio y que resulta de la relación dialéctica entre el medio y las acciones sociales” (Fontecilla, 1998, p. 9), mucho debe decirnos el análisis crítico de su anatomía para develar lo que Arreola y Curtis (1993) denominan como "la personalidad" del lugar. En efecto, una aproximación superficial al análisis del paisaje -y por tanto, a la interacción entre las determinantes estructurales y la acción de los sujetos en la formación de los espacios particulares- revela una localidad que si bien no es predominantemente agrícola, acusa algunos atributos que la dotan de un fuerte carácter rural.

Las estadísticas también rinden cuenta de ello. De acuerdo con el censo del año 2000 la población ocupada de la localidad era de 1783 personas, de las cuales $69 \%$ laboraba en el sector secundario, $22 \%$ en el sector terciario y $8 \%$ en el sector primario. 
Sin embargo, al analizar de manera minuciosa las diferentes categorías bajo las cuales se insertaban al mercado de trabajo los habitantes de San Diego Cuachayotla, es posible concluir que la gran mayoría desarrollaba su actividad laboral dentro de la comunidad al trabajar por cuenta propia, tanto en la fabricación de tabique, como en la agricultura (54.61\%), o al vender su fuerza de trabajo como jornalero o peón (20.3\%). El resto (25\%) se ocupaba como empleado u obrero en localidades lindantes.

El carácter familiar, propio de las actividades agrícolas y de la producción de ladrillo, se puede constatar en el número de personas que trabajaban sin percibir ingresos (30.II\%) o que recibían menos de un salario mínimo mensual como retribución por su trabajo (16\%). Asimismo, la inseguridad laboral y la falta de prestaciones se aprecia en el porcentaje de la población sin derecho a servicios de salud, la cual alcanzaba para ese año poco más de 92\%3 (INEGI, 2000). De esta manera, tras el sesgo industrial que muestran las estadísticas, se esconde el desarrollo de una actividad cuyo proceso de organización y producción corre a cargo de las familias y que, por tanto, no guarda similitud alguna con la pequeña empresa capitalista que domina los escenarios urbanos. De igual forma, se encubre o minimiza el peso de la actividad agrícola, que si bien ha perdido importancia relativa a lo largo de las últimas décadas, mantiene su desarrollo en San Diego Cuachayotla, fundamentalmente en el ámbito del traspatio.

Como veremos a lo largo de este artículo, la estructura social y productiva de esta localidad, así como las posiciones relativas de los agentes que la habitan, han cambiado de manera significativa en las últimas décadas. Este cambio resultado, entre otras cosas, de la dinámica de crecimiento urbano- ha afectado la configuración del espacio social de San Diego Cuachayotla, lo que ha modificado la composición patrimonial de los grupos domésticos periurbanos, e incidido, por ende, en la formación de nuevas estrategias reproductivas.

\section{El repliegue de la actividad agrícola}

La zona donde se ubica San Diego era, desde antes de la llegada de los españoles, un importante centro agrícola. Sin embargo, con el paso del tiempo, este carácter de la localidad se ha ido transformando.

3 El censo de 2010 no incluyó ninguno de los indicadores anteriormente señalados, lo que impide dar un seguimiento puntual a la evolución de la estructura productiva en San Diego Cuachayotla. 
De acuerdo con los testimonios de los habitantes de la región, a mediados del siglo pasado se sembraban y cosechaban en San Diego Cuachayotla, además de cultivos nativos como el maíz, el maguey, el chile y el frijol, otros cultivos introducidos por los españoles como los frutales y el trigo. Estos testimonios coinciden con las descripciones que hacen algunos cronistas de la Conquista (Díaz, 1976) y de la Colonia (De Rojas, 1979) en diversos documentos históricos, lo que de alguna manera apunta a que el tipo de agricultura practicada en esta región se mantuvo casi sin cambios durante, por lo menos, 400 años.

La alteración de la estructura agraria, acaecida a principios del siglo pasado tras el periodo revolucionario, y la adopción de un modelo económico que subordinó la agricultura y el trabajo campesino a las necesidades de la industria y del crecimiento urbano, suscitaron cambios importantes en las prácticas agrícolas en San Diego Cuachayotla. Los propios habitantes de esta localidad identifican dos procesos que han incidido de manera importante en la transformación de la estructura productiva: la pulverización de la propiedad agrícola y el dinamismo de la actividad ladrillera.

En cuanto a lo primero, valga decir que la presión demográfica se ha convertido en el factor primordial de la excesiva fragmentación de la propiedad privada agrícola. En el caso de San Diego Cuachayotla, como en gran parte de las localidades rurales de México, la herencia no es solo un patrimonio económico, sino un legado que el jefe de familia está obligado moralmente a transmitir a sus hijos. La costumbre de heredar los terrenos agrícolas a todos los descendientes directos de un grupo doméstico, ha contribuido a hacer del minifundio la forma generalizada que asume hoy en día la propiedad de la tierra.

La multiplicación del número de predios de una generación a otra, aunada a la imposibilidad de extender el área agrícola más allá de los límites propios de la localidad, ha propiciado a lo largo del tiempo un cambio significativo en su estructura productiva. Si consideramos además que en San Diego Cuachayotla se carece de la infraestructura adecuada para el desarrollo de una agricultura de riego, no es de extrañar que una actividad más rentable como la producción de ladrillo, haya desplazado desde hace ya varias décadas a la agricultura como la principal fuente de ingresos.

A diferencia del pasado, la agricultura no es hoy la actividad preponderante entre los habitantes de San Diego Cuachayotla. La información que reporta el Consejo Municipal de Desarrollo Rural Sustentable (CMDRS, 2008) ubica la cadena maíz como la única que se cultiva en esta junta auxiliar. De acuerdo con este organismo, la superficie de maíz criollo sembrado es de 60 hectáreas, es decir, $20 \%$ de la 
superficie total de la localidad. Dadas las condiciones bajo las cuales se cultiva este grano, el rendimiento medio alcanza apenas las dos toneladas por hectárea. Por otra parte, se ha identificado de manera oficial a 300 productores de maíz en esta zona.

En cuanto a la ganadería, se ha registrado un total de I20 productores que, en su conjunto, cuentan con 400 cabezas de ganado vacuno destinado a la producción de leche y sus derivados, tanto para el autoconsumo, como para la comercialización en el mercado local. Existen también 150 productores que poseen en su conjunto cerca de 450 cerdos y 65 productores que se dedican a la crianza de 500 cabezas de ganado ovino, las cuales se comercializan en los mercados locales y regionales, y cuyo consumo forma parte de la dieta de los grupos domésticos de la región. Cabe agregar que en todos los casos la ganadería constituye una actividad limitada al traspatio y que, más que un negocio, implica una estrategia productiva orientada al ahorro, ya sea con fines de autoconsumo o para hacer frente a alguna eventualidad de tipo económica.

Pese al papel marginal que otorgan estas cifras a la agricultura, la gran mayoría de los pobladores de esta localidad mantiene el cultivo de maíz, aunque en espacios reducidos que incluyen el traspatio y otras fracciones de terrenos que se ubican dentro o fuera del pueblo. Este maíz no se comercializa; se destina para el autoconsumo del grupo doméstico y para la alimentación de los animales. Así, el maíz continúa como elemento cultural importante que no solo compone la base de la alimentación, sino que representa además una garantía de supervivencia para los grupos domésticos, que se ven obligados a adquirir el resto de los alimentos que integran su dieta en el mercado.

Cabe señalar, con base en las entrevistas en profundidad realizadas en esta localidad, que existe una divergencia notable entre la orientación del discurso y las actitudes de los pobladores de San Diego Cuachayotla hacia la actividad agrícola. Por un lado, los habitantes se muestran escépticos con respecto a la continuidad de las prácticas agrícolas en su localidad: el minifundismo, el alto precio de los insumos y la poca rentabilidad de la agricultura con respecto a otras actividades, se mencionan de modo constante como factores que operan en contra del desarrollo de la actividad agrícola.

Lo curioso es que al tiempo que se sostiene un discurso que, en apariencia, niega toda posibilidad a la agricultura, existe un número considerable de pobladores que pese a las condiciones económicas adversas insiste en cultivar la tierra. Esto, al punto de canalizar recursos provenientes de actividades más redituables, como la producción de ladrillo, para adquirir terrenos en las localidades cercanas o financiar las prácticas agrícolas. De igual forma, pese a las limitantes que imponen los 
condicionamientos sociales externos a las prácticas agrícolas en esta localidad, los entrevistados consideran que la enseñanza de la agricultura forma parte de los conocimientos -es decir, del patrimonio simbólico y cultural- que se deben transmitir a las generaciones futuras.

Tal persistencia deja al descubierto el papel que desempeña en la producción de las prácticas la noción de “interés”, entendida no en su acepción economicista, sino en un sentido más amplio, como un interés socialmente constituido en relación con un espacio social concreto, en el seno del cual ciertas cosas son importantes y otras indiferentes (Bourdieu, 1994). Así, para los habitantes de San Diego Cuachayotla, las prácticas agrícolas forman parte de los intereses específicos que se ponderan más allá de su vertiente económica.

\section{La producción de ladrillo, eje de las estrategias reproductivas}

Como se ha señalado, existen ciertas características de orden estructural, intrínsecas a esta localidad, que imponen restricciones a la producción agrícola y que, en confluencia con otros procesos, han generado una estructura productiva afincada en el desarrollo de la actividad ladrillera. Sin embargo, cabe señalar que en San Diego Cuachayotla la producción de ladrillo no constituye de ninguna manera una actividad impuesta desde el exterior a los pobladores locales. Por el contrario, al igual que la agricultura, se trata de una actividad que se encuentra históricamente arraigada en el territorio cholulteca4.

El cronista del periodo de Conquista, Bernal Díaz del Castillo, afirmó que desde la época prehispánica, Cholula era famosa por su alfarería ya que se hacía "muy buena loza de barro, colorado e prieto, e blanco, de diversas pinturas, e se abastece della México e todas las provincias comarcanas, digamos ahora como en Castilla lo de Talavera o de Palencia” (en Bonfil, I988, p. 75). En otro pasaje, el cronista narra cómo la producción de cerámica de esta región era tan valorada que incluso el propio Moctezuma tenía una vajilla de origen cholulteca para ocasiones especiales (en Ruz Barrio, 2008, p. 17).

4 El uso de ladrillo como elemento de construcción tuvo su apogeo en Cholula durante el periodo que comprende de los años 200 d.C. al 700 d.C. 
Tras la aniquilación de los indígenas, la famosa y fina cerámica de Cholula desapareció y dejó como evidencia de su antiguo esplendor, la producción a pequeña escala de comales, objetos simples de manufactura burda que todavía hasta mediados del siglo pasado se producían en localidades como San Diego Cuachayotla y se comercializaban en los tianguis locales. Hoy en día, la producción de comales ya no existe, pero el barro con el cual se fabricaban es aún materia prima importante en la fabricación de ladrillos para la construcción.

De acuerdo con los testimonios de los habitantes de San Diego, la fabricación de ladrillo empezó a cobrar importancia a mediados del siglo pasado, a partir de la demanda suscitada por el ritmo de urbanización de las grandes ciudades, en especial, la ciudad de México. En aquellos años, las ladrilleras de Cholula recibían pedidos en tales cantidades, no solo de Puebla sino también de la capital del país y otros estados vecinos, que en determinadas épocas del año superaban la capacidad de producción local.

El auge de la industria ladrillera -que tuvo su apogeo en los años sesentay de los beneficios económicos derivados de esta actividad, tuvo como consecuencia directa que la mayor parte de la población de San Diego Cuachayotla incursionara en la producción de ladrillo. Así, en el curso de unos cuantos años los hornos se multiplicaron en esta localidad y en las circundantes de manera sorprendente (Ashwell, 2005) 5 .

Para la población de San Diego, el cambio de actividad significó también -en sus propias palabras- "un cambio de vida”. De hecho, la acumulación de capital económico durante este periodo es uno de los temas más recurrentes en los testimonios de los pobladores más viejos, a quienes les tocó experimentar el cambio de un modo de vida afincado en el desarrollo de las prácticas agrícolas, a otro donde la producción de ladrillo constituía el eje de la vida económica y social de la región. De esta manera, la fabricación de ladrillo desplazó de forma paulatina a la agricultura, pasó de ser una actividad complementaria a convertirse en la principal -y en algunas ocasiones, la única- fuente de ingresos de los grupo domésticos.

Actualmente son 470 las familias de San Diego que se dedican a esta actividad. Asimismo se tienen ubicados un total de 322 hornos, cada uno de los cuales produce un promedio de 30000 ladrillos al mes. Datos obtenidos a partir de una

5 De acuerdo con esta autora, a principios de los años noventa se censaron en el municipio de San Pedro Cholula, 5218 familias ladrilleras, las cuales era dueñas de I786 hornos, ubicados en las localidades San Diego Cuachayotla, San Juan Tlautla, San Sebastián Tepalcatepec, San Cosme Texitla, San Matías Cocoyotla, San Cristóbal Tepontla, Santa Bárbara Almoloya y San Francisco Coapa. 
encuesta reciente (Xamixtli, 2006) señalan que la sobrevivencia de más de $75 \%$ de la población económicamente activa depende, directa o indirectamente, de la producción de ladrillo.

La tecnología con que se lleva a cabo la fabricación de tabique en la actualidad, demanda una gran cantidad de fuerza de trabajo, concentrada además en cierta época del año, lo que impone ciertos requerimientos en términos de organización familiar y de uso de recursos, que inciden en el desarrollo de estrategias de reproducción específicas para los grupos domésticos de San Diego.

En este punto, conviene hacer referencia a las diferencias que guardan los grupos domésticos de San Diego en relación con algunas de las características que la literatura atribuye a los grupos domésticos campesinos, en particular las que se desprenden de la naturaleza del valor producido en ambos tipos de unidades (Margulis, 1989; Pepin Lehalleur y Rendón, 1989; De Oliveira y Salles, 1989; Giner de los Ríos, 1989).

Un aspecto crucial que define la configuración -y por tanto las estrategias de reproducción- de los grupos domésticos de San Diego Cuachayotla, es el peso que tiene la actividad ladrillera en el ingreso de las familias. Este factor, aunado a la forma de organización laboral que priva en los espacios reservados para la producción de ladrillo, impone una modalidad de funcionamiento bajo la cual los grupos domésticos se organizan como "empresas familiares puras" (Giner de los Ríos, 1989). En estas empresas familiares el trabajo y la propiedad de los medios de producción son los recursos del grupo doméstico para asegurar un flujo de ingresos constante que garantice la reproducción de sus integrantes, objetivo distinto al de la pequeña empresa capitalista, cuyo propósito es la obtención de ganancia a partir de la apropiación del trabajo impago implícito en la relación salarial.

La conjugación en una sola entidad de estos dos principios de agrupación y de funcionamiento, es la que confiere a los grupos domésticos de San Diego características, necesidades y posibilidades intrínsecas, distintas a las que se observan en los grupos domésticos de base campesina, los cuales pueden tratar los productos como valores de cambio o como valores de uso según su destino (Cook y Binford, 1995).

En el caso de los grupos domésticos de San Diego Cuachayotla el acento está puesto fundamentalmente en la producción de valores de cambio. De ahí que para esta localidad, el mercado constituya una instancia más crucial para la reproducción, desde el punto de vista comparativo, ya que a diferencia de las unidades campesinas típicas, esta depende de la venta de productos que por su propia naturaleza no pueden ser autoconsumidos. 
Por otra parte, la producción de ladrillo supone la concentración de fuerza de trabajo en algunos periodos, mientras que en otros, la cantidad requerida se reduce al mínimo. Por lo general, los periodos de alta demanda están vinculados con las diversas etapas del proceso productivo, con los vaivenes del mercado regional y sobre todo, con las condiciones climatológicas que impiden la elaboración de tabique en ciertas épocas del año. Con base en estos factores se organizan los tiempos dedicados a otras actividades productivas. Así, para las familias de San Diego Cuachayotla es el proceso de producción de ladrillo -y no el ciclo agrícola, como sucede en los grupos domésticos campesinos- el factor que determina y condiciona el resto de las actividades.

Los requerimientos discontinuos de trabajo que caracterizan la producción de ladrillo en San Diego Cuachayotla crean espacios para la diversificación de labores, y en particular, para el desarrollo de las actividades agrícolas. El hecho de que el tipo de agricultura que se practica en esta localidad sea de temporal, la convierte en una actividad que se puede desarrollar a intervalos, durante los periodos en que la producción de tabique se suspende o disminuye de intensidad. En el caso de los grupos domésticos que carecen de tierra, es frecuente que las labores propias de la actividad ladrillera se intercalen con breves periodos durante los cuales alguno de los miembros de la familia ofrece su fuerza de trabajo a cambio de una remuneración económica, al buscar ser contratado como jornalero o peón en las poblaciones vecinas.

Pese al predominio del binomio "producción ladrillera-agricultura" en la composición de las ocupaciones desarrolladas por los grupos domésticos entrevistados, cabe señalar que también se suelen presentar algunos casos en que la fabricación de tabique coexiste con otro tipo de actividades, ya sea de índole comercial o de servicios, que se desarrollan dentro o fuera de la localidad.

\section{La organización familiar de los grupos domésticos periurbanos}

Diversos autores que han realizado investigaciones sobre las dinámicas de organización interna de las familias (Chayanov, 1974; González de la Rocha, 1986; Salles, 1989), refieren que uno de los elementos que determina de manera más directa a cuál actividad o actividades particulares se consagrará el grupo doméstico, es la etapa del ciclo familiar por la cual transita. Esta etapa determina la magnitud y composición de la fuerza de trabajo disponible para organizar y distribuir, tanto el tiempo como las actividades productivas susceptibles de desarrollarse. 
Las entrevistas que se realizaron durante el trabajo de campo en San Diego Cuachayotla permiten confirmar que la morfología de la familia, su tamaño, edad de los miembros y los tipos de actividad para los cuales son aptos, constituyen los elementos inmediatos que determinan la organización del trabajo en el interior de cada uno de estos grupos domésticos.

En particular, un aspecto relevante es que, dada la carga física que demanda la fabricación de ladrillo, la presencia mano de obra adulta masculina constituye un requisito indispensable para la incursión del grupo doméstico en esta actividad. Este factor se configura como una suerte de "barrera a la entrada" que limita la participación de los grupos locales que carecen de este recurso. De esta manera, los grupos domésticos con jefatura femenina que no tienen posibilidades de contratar fuerza de trabajo adicional para la producción de tabique o para el desarrollo de actividades agrícolas, se ven obligados a incursionar en otro tipo de actividades, por lo regular, asociadas con el comercio o con la prestación de servicios a otros grupos domésticos a cambio de una remuneración económica o en especie.

Es así como los grupos domésticos que cuentan con el padre y varios hijos varones, tienen un mayor número de opciones para organizar y distribuir actividades; en tanto que los grupos que, por condición de familia o por la migración de algunos de sus miembros, carecen de mano de obra masculina, dependen necesariamente de la contratación de jornaleros o peones para intervenir en el monto de trabajo disponible y contrarrestar la rigidez impuesta por la estructura original de la familia.

Si bien la mano de obra masculina es una condición básica para el desarrollo de la actividad ladrillera, es necesario enfatizar que la mayor parte de las veces este requisito, por sí mismo, no es suficiente para cubrir la cuota de trabajo físico que esta labor implica. Por tal motivo, el involucramiento de todos los integrantes del grupo doméstico en el proceso productivo constituye un factor común en los talleres de San Diego, donde sin distinción de género o generación, cada uno de los miembros de las familias aporta fuerza de trabajo en la medida que sus condiciones lo permiten. De esta manera, el carácter familiar del grupo doméstico se convierte en un recurso fundamental para superar las limitaciones inherentes a un monto de fuerza de trabajo, predeterminado independientemente de las necesidades particulares del grupo en un momento dado.

Pepin Lehalleur y Rendón (1989) sostienen que la estrecha vinculación entre miembros de generaciones diferentes en el proceso mismo de trabajo, permite a los grupos domésticos campesinos aprovechar la mano de obra disponible y establecer condiciones de complementariedad y cooperación en múltiples actividades. 
La forma en que los grupos domésticos periurbanos de San Diego Cuachayotla distribuyen los tiempos y la intensidad de las labores cotidianas entre sus miembros, coincide con la observación de estas autoras en términos de que las estrategias que desarrollan implican no solo la utilización intensiva de mano de obra familiar, sino la maximización de la fuerza de trabajo disponible -fundamentalmente bajo relaciones de tipo no capitalista-, como un mecanismo para suplir la falta de garantías externas para la reproducción.

De esta forma, ciertas modalidades de trabajo que en un contexto urbano son poco frecuentes, o que por lo menos se encuentran sancionadas socialmente, forman parte de la cotidianidad de los grupos domésticos de San Diego Cuachayotla. Tal es el caso del trabajo infantil, el cual además de desempeñar un papel central en la transmisión de conocimiento, constituye una fuente muy importante, aunque temporal, de fuerza de trabajo.

Asimismo, no es raro que las mujeres sean las que más trabajo aportan al grupo doméstico, ya que aparte de compartir la responsabilidad del trabajo productivo tanto en el campo como en la producción de ladrillo, están a cargo de las labores reproductivas. Por lo general, se trata de actividades que no pueden cuantificarse en términos monetarios porque no hay mediación del mercado, ya que los miembros de las familias funcionan al mismo tiempo como productores y consumidores de las mismas (Salles, 1989). Es usual que estas responsabilidades recaigan en las madres o hijas a partir de cierta edad, y pueden concebirse en el marco de las estrategias de intensificación y diversificación del trabajo (Pepin Lehalleur y Rendón, 1989).

La estrategia de utilización intensiva de la mano de obra familiar permite a los grupos domésticos prescindir de la contratación de fuerza de trabajo adicional, un factor que -dado el contexto económico adverso en el cual se desarrollan actualmente tanto la actividad ladrillera, como la agricultura- harían inviables cualquiera de estas prácticas.

\section{Una localidad en proceso de cambio}

Una primera conclusión que se desprende del análisis de las estrategias de reproducción social ligadas al capital económico en San Diego Cuachayotla, es que el ingreso de los grupos domésticos -y por tanto las condiciones que favorecen la reproducción en esta localidad- dependen en gran medida de la cantidad de trabajo familiar disponible, la cual está determinada por el número de integrantes del grupo 
y por la composición genérica y etaria del mismo, es decir, por la etapa específica del ciclo doméstico en que este transita. Al respecto, la situación de los grupos domésticos en San Diego Cuachayotla coincide con la que Pepin Lehalleur y Rendón (ı989, p. I23) describen en el estudio realizado en tres comunidades campesinas de distintas regiones de México, donde encontraron que los grupos domésticos con mayor acceso relativo a recursos productivos y con niveles de vida relativamente mejores son siempre grandes: "Cuentan con muchos trabajadores y un número excepcionalmente elevado de consumidores lo que indica que en esas condiciones de acceso a los medios, disponer de fuerza de trabajo abundante permite no solo aumentar la producción sino también la retribución por jornada familiar y aun por consumidor”.

También comparte similitudes con los resultados de la investigación que realizó Margulis (1989) sobre el comportamiento reproductivo de las unidades domésticas urbanas de tipo intermedio, en la cual se concluye que el bienestar de estas unidades está ligado a la relación interna entre productores de ingresos y consumidores de ingresos, lo que a su vez dependería de los momentos del ciclo biológico familiar en que tal relación es favorable.

Al igual que los grupos descritos por estos investigadores, los grupos domésticos de San Diego se ven obligados a desempeñar actividades múltiples y aumentar el número de jornadas que despliega la familia en su conjunto. Este es el único camino disponible para hacer frente a las necesidades crecientes o para mejorar las condiciones de su reproducción y por ende, su posición en el espacio social.

La reconfiguración de las estrategias reproductivas en San Diego Cuachayotla ha implicado el surgimiento de nuevas actividades económicas; la intensificación de algunos fenómenos sociales, como la migración y la pluriactividad; así como la adopción progresiva de patrones culturales e identitarios inéditos.

En la actualidad, el contexto económico en el cual tiene lugar la producción de ladrillo es sumamente adverso. El encarecimiento de las materias primas -sobre todo, el petróleo-, aunado a la sobreoferta de producto en la región y la contracción del sector construcción, han cancelado la posibilidad de mejorar la posición de los grupos domésticos en el espacio social mediante la intensificación del trabajo familiar. Esta situación pone en duda hoy la viabilidad de la producción de ladrillo como una actividad productiva, capaz de generar los recursos económicos que aseguren la reproducción de los grupos domésticos de San Diego ${ }^{6}$.

6 Para 2005, por ejemplo, el costo promedio de un millar de ladrillo era de 800 pesos, aunque el precio de mercado llegó a bajar durante ese mismo año hasta los 500 pesos por millar. 
A las condiciones económicas desfavorables intrínsecas a la producción de ladrillo se suman otros factores, como el crecimiento urbano y el incremento de los flujos migratorios, que han abierto el abanico de oportunidades para que las generaciones más jóvenes incursionen en actividades laborales fuera de la localidad, ya sea en la ciudad de Puebla, en el Distrito Federal o incluso fuera del país. No es extraño que en este contexto la escolaridad se haya convertido en una prioridad para los grupos domésticos de San Diego Cuachayotla, cuyos integrantes perciben que la obtención de un título que acredite un nivel de formación superior, garantiza a los hijos el acceso, en condiciones más favorables, al mercado de trabajo. Esto constituye actualmente el instrumento de reproducción que ofrece mayor rendimiento a las inversiones de los agentes sociales (Hernández, 20ro).

Finalmente en lo que concierne al papel de la agricultura, es necesario mencionar que pese a que en la actualidad esta se concibe como una actividad complementaria, desarrollada a tiempo parcial, que se integra por lo regular a otras actividades generadoras de ingreso, como la producción de ladrillo o la migración, para los habitantes de San Diego Cuachayotla es todavía una práctica relevante, muy arraigada. La agricultura responde a consideraciones que superan cualquier racionalidad de índole económica, ya que se encuentra asociada con la persistencia de un modo de vida del cual no quieren desprenderse.

En este sentido, se pudo constatar que en San Diego Cuachayotla la agricultura desempeña, además de una importante función en la recreación de la identidad campesina, al menos otras tres importantes funciones: a) reducción de la inseguridad alimentaria; b) uso productivo de recursos disponibles y no utilizados; y c) una importante fuente de ahorro, debido a que permite canalizar a otros usos, los recursos monetarios que de otro modo tendrían que destinar a la compra de alimentos.

\section{Conclusiones}

Las periferias de las ciudades, en su calidad de zona de contacto entre los mundos rural y urbano, constituyen el marco en el cual se desarrollan complejos procesos territoriales y donde acontecen importantes disputas por el uso del espacio. La conflictividad propia de estos territorios hace necesario conocer las dinámicas productivas y las lógicas territoriales con las cuales operan los diferentes agentes sociales, con la intención de normar algunos de los procesos mediante políticas y estrategias orientadas al ordenamiento de estos ámbitos (Ávila, 2009). La tarea no es 
asunto sencillo; sin embargo, conocer la forma en que los grupos domésticos de estas localidades se reproducen socialmente puede arrojar evidencia a partir de la cual se pueden definir líneas de acción, priorizar tareas, o incluso, descartar intervenciones.

Para el caso de la localidad analizada, por ejemplo, cualquier intervención que busque mejorar las condiciones de vida de sus habitantes, debería reconocer el importante papel que desempeñan las prácticas agrícolas en las estrategias de reproducción social de los grupos domésticos. Ante el crecimiento inminente de la ciudad y las posibilidades limitadas de la población rural para insertarse con éxito en los procesos urbanos, la agricultura puede representar una vía para el fortalecimiento de la seguridad alimentaria de los grupos domésticos, al tiempo de posibilitar la pluriactividad y la diversificación del ingreso familiar.

Ante la reconfiguración productiva que experimentan los territorios periurbanos y la consiguiente pérdida de centralidad de las actividades agrícolas, es necesario retomar, como piedra angular de cualquier estrategia de intervención, un concepto ampliado de desarrollo rural que incluya el conjunto de actividades actuales y potenciales, rurales y no rurales, importantes para la construcción de medios de subsistencia sostenibles. En este sentido, resulta prioritario emprender políticas para la generación de oportunidades de empleo en otros sectores y en otros ámbitos que contribuyan a complementar el ingreso de las familias.

\section{Referencias}

Arias, P. (2006). Migración, familia y herencia en el campo mexicano. Recuperado el 23 de febrero de 2012, de http://estudiosdeldesarrollo.net/coloquiozoo6/ documentos/29182.doc

Arreola, D. y Curtis, J. (1993). The Mexican Border Cities. Landscape Anatomy and Place Personality. Tucson: University of Arizona Press.

Ashwell, A. (2005) El Tzapotecas es de los cholultecas. La Jornada (3 de agosto de 2005). Ávila, H. (200I). Ideas y planteamientos teóricos sobe los territorios periurbanos. Las relaciones campo-ciudad en algunos países de Europa y América. Revista Investigaciones Geográficas, (45), 108-127.

Ávila, H. (2004). La agricultura en las ciudades y su periferia: un enfoque desde la geografía. Revista Investigaciones Geográficas, (53), 98-I2I.

Ávila, H. (2009). Periurbanización y espacios rurales en la periferia de las ciudades. Revista Estudios Agrarios, (4I), 93-II8. 
Banzo, M. (2005). Del espacio al modo de vida. La cuestión periurbana en Europa occidental: los casos de Francia y España. En H. Ávila (Comp.), Lo urbano-rural, ¿nuevas expresiones territoriales? (pp. 207-244). México D. F.: UNAM-CRIM.

Barsky, A. (2005). El periurbano productivo, un espacio en constante transformación. Introducción al estado del debate, con referencias al caso de Buenos Aires. Scripta Nova, Revista Electrónica de Geografía y Ciencias Sociales, IX (194). Recuperado el 31 de marzo de 20I4, de http://www.ub.edu/geocrit/sn/sn-194-36.htm

Bonfil, G. (1988). Cholula, la cindad sagrada en la era industrial. Puebla: Universidad Autónoma de Puebla.

Bourdieu, P. (1994). ¿Qué es lo que hace una clase? Acerca de la existencia teórica y práctica de los grupos. Revista Paraguaya de Sociología, 3I (89), 7-2I.

Chayanov, A. (1974). La organización de la unidad doméstica campesina. Buenos Aires: Ediciones Nueva Visión.

Concejo Municipal de Desarrollo Rural Sustentable (2008). Identificación de cadenas agropecuarias en San Pedro Cholula. Puebla: Autor.

Cook, S. y Binford, L. (1995). La necesidad obliga. La pequeña industria rural en el capitalismo mexicano. México D. F.: Consejo Nacional para la Cultura y las Artes.

De Janvry, A. y Sadoulet, E. (200I). Income Strategies among Rural Households in Mexico: the Role of Off-Farm Activities. World Development, 29(3), 467-480.

De Oliveira, O. y Salles, V. (1989). Acerca del estudio de los grupos domésticos: un enfoque sociodemográfico. En O. de Oliveira et al. (Coords.), Grupos domésticos y reproducción cotidiana (pp. II-36). México D. F.: Colmex-UNAM.

De Rojas, G. (1979). Descripción de Cholula. Puebla: Gobierno del estado de Puebla. Delgado, J. (2003). Transición rural-urbana y oposición campo-ciudad. En A. Aguilar (Coord.), Urbanización, cambio tecnológico y costo social. El caso de la región centro de México (pp. 73-118). México D. F.: UNAM, Conacyt, Miguel Ángel Porrúa. Díaz del Castillo, B. (1976). Historia verdadera de la conquista de la Nueva España. México D. F.: Miguel Ángel Porrúa.

Dirven, M. (2004). El empleo rural no agrícola y la diversidad rural. Revista de la Cepal, (63), 49-68.

Entrena, F. (2005). Procesos de periurbanización y cambios en los modelos de ciudad. Un estudio sobre sus causas y consecuencias. Papers, (78), 59-88.

FAO (1999). La agricultura urbana y periurbana. Comité de Agricultura, XV periodo de sesiones. Recuperado el 23 de febrero de 20I2, de http://www.fao.org/unfao/ bodies/coag/coag15/xoo76s.htm 
Fontecilla, A. (1998). Calidad de lo urbano: representaciones sociales. Revista Ciudades, RNIU, (38), I4-18.

Galimberti, S. (20II). Rurbanidad, objetos y significaciones. Un estudio acerca de los actores rurbanos y la política pública. Ponencia presentada en XXXIV Congresso Brasileiro de Ciencias da Comunicação. Recife: Intercom-Sociedade Brasileira e Estudos Interdisciplinares da Comunicação.

Giner de los Ríos, F. (1989). Microindustria y unidad doméstica. En O. de Oliveira et al. (Coords.), Grupos domésticos y reproducción cotidiana. México D. F.: Colmex-UNAM.

González de la Rocha, M. (1986). Los recursos de la pobreza. Familias de bajos ingresos de Guadalajara. México D. F.: El Colegio de Jalisco, Ciesas, SPP.

Hernández, J. (2010). Estrategias de reproducción social en grupos domésticos periurbanos. Un estudio comparativo en tres localidades poblanas. (Tesis de doctorado no publicada) Colegio de Postgraduados. Programa de Estrategias para el Desarrollo Agrícola Regional. Puebla, México. Recuperado el 3i de marzo de 20I4, de http://www. biblio.colpos.mx:8080/xmlui/handle/1052I/135

Hernández, J. y Méndez, J. (2007). Nueva ruralidad y nuevos actores sociales en el medio rural. Evidencias empíricas para México. En Estudios y propuestas para el medio rural, Tomo II. Sinaloa: Colegio de Postgraduados-UAIM.

Instituto Nacional de Estadística y Geografía [INEGI] (2000). Censo general de población y vivienda. Aguascalientes: Autor.

Inegi (2010). Censo general de población y vivienda. Aguascalientes: Autor.

Kay, C. (2004). Las políticas agrarias en Europa y en América Latina. En E. Pérez (Comp.), Desarrollo rural y nueva ruralidad en América Latina y la Unión Europea (pp. 8I-90). Bogotá D.C.: Pontificia Universidad Javeriana.

Klein, E. (1992). El empleo rural no agrícola en América Latina. Documento de trabajo 364. Santiago: Prealc.

Margulis, M. (1989). Reproducción de la unidad doméstica, fuerza de trabajo y reproducción. En O. de Oliveira et al. (Coords.), Grupos domésticos y reproducción cotidiana (pp. 189-215). México D. F.: Colmex-UNAM.

Navarro, H. (2005). Transformaciones de los territorios periurbanos y sus agriculturas: el uso de recursos de interés público en el valle de México. En H. Ávila (Coord.), Lo urbano-rural, ¿nuevas expresiones territoriales? (pp. 245-275). Morelos: Centro Regional de Investigaciones Multidisciplinarias-UNAM. Pepin Lehalleur, M. y Rendón, T. (1989). Reflexiones a partir de una investigación sobre grupos domésticos campesinos y sus estrategias de reproducción. En O. 
de Oliveira et al. (Coords.), Grupos domésticos y reproducción cotidiana (pp. 107-125). México D.F.: Colmex-UNAM.

Pérez, E. (200I). Hacia una nueva visión de lo rural. En N. Guiarraca (Comp.), ¿̇Una nueva ruralidad en América Latina? (pp. 15-29). Buenos Aires: Clacso.

Pérez, R. (2005). Las transformaciones de la estructura agraria ejidal en la zona conurbada de la ciudad de Puebla (1980-2003). En R. Pérez, S. Gómez y H. Ávila (Coords.), Lo urbano desde lo rural. El caso de la zona conurbada de la ciudad de Puebla (1980-2004) (pp. 50-III). Puebla: BUAP.

Ramírez, C. (2006). Crítica al enfoque del desarrollo territorial rural. Revista ALASRU, Nueva época, (3), 49-80.

Reardon, T., Berdegué, J. y Escobar, G. (200I). Rural Non-Farm Employment and Incomes in Latin America: Overview and Policy Implications. World Development, 29(3), 395-409.

Rubio, B. (2006). Una teoría con campesinos: los despojados del nuevo imperialismo. Revista ALASRU, nueva época, (3), 8I-102.

Ruz, M. (2008). Cholula durante el siglo XVI: la familia Chimaltecuhtli-Casco. Revista Española de Antropología Americana, 38(I), 7-29.

Salles, V. (1989) Una discusión sobre las condiciones de la reproducción campesina. En O. de Oliveira et al. (Coords.), Grupos domésticos y reproducción cotidiana (pp. I27-159). México D.F.: Colmex-UNAM.

Xamixtli (2006). Alternativas para la cocción de ladrillo en San Diego Cuachayotla Cholula. Reporte interno. Puebla: Autor. 